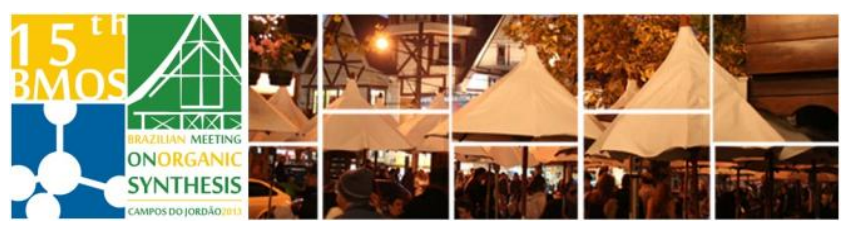

\title{
3,4-(methylenodioxy)aniline as precursor to thiazolidinones
}

\section{Hellen G. Masteloto (PG), Bruna B. Drawanz (PG), Geonir M. Siqueira (PQ), Wilson Cunico*(PQ)}

LaQuiABio,CCQFA, UFPel, Pelotas, RS, Brazil

*wjcunico@yahoo.com.br

Keywords: Thiazolidinones, 3,4-(methylenedioxy)aniline, one-pot reaction

\section{INTRODUCTION}

The thiazolidinones are five-membered heterocyclic compounds that show a diverse range of biological activities ${ }^{1}$, for example, as antitumor ${ }^{2}$, antidiabetes $^{3}$, antitubercular ${ }^{4}$ and anti-hepatitis $C$ virus ${ }^{5}$. The main synthetic routes to thiazolidin-4ones involves a three component reaction (an aldehyde or ketone, a primary amine or hydrazine and the mercaptoacetic acid) either in an one- or two-step process. ${ }^{6}$ This work has as objective, report the synthesis of new thiazolidinones 4a-r arising from the reaction of 3,4-(methylenedioxy)aniline 1 with substituted arenealdehydes 2a-r and mercaptoacetic acid 3.

\section{RESULTS AND DISCUSSION}

The synthesis of unpublished thiazolidinones 4a-r, was carried out in a one-pot procedure (Scheme 1). First, the reaction of amine 1 (1 $\mathrm{mmol})$ with arenealdehydes 2a-r $(1 \mathrm{mmol})$ in toluene reflux using a Dean-Stark trap for $3 \mathrm{~h}$ afforded the imine intermediate. Afterward, the mercaptoacetic acid 3 ( $3 \mathrm{mmol}$ ) was added and the reaction progress were monitored by thin layer chromatography (TLC) and/or Gas Chromatography (GC). The products were formed after overnight reflux and the pure thiazolidinones were obtained by washing with a hot solution of hexane/ethyl acetate 9:1 (compounds 4aI) and 8:2 (compounds $4 \mathrm{~m}-\mathbf{r}$ ) from good to excellent yields $47-90 \%$ (Table 1). All compound structures were confirmed by mass spectrometry (CG-MS), ${ }^{1} \mathrm{H}$ and ${ }^{13} \mathrm{C}$ Nuclear Magnetic Resonance (NMR)

Scheme 1. Synthesis thiazolidinones 4a-r

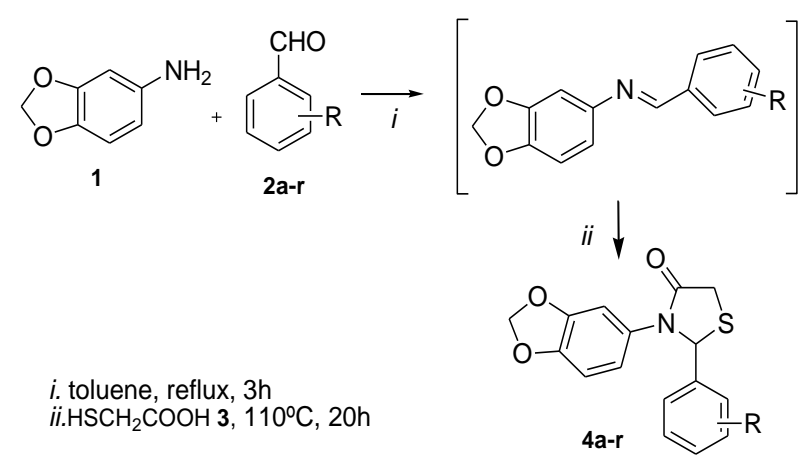

Table 1. Yields and melting points of thiazolidinones 4a-r.

\begin{tabular}{|c|c|c|c|}
\hline Product & $\mathbf{R}$ & yield (\%) $^{\mathbf{a}}$ & m.p. $\left({ }^{\circ} \mathbf{C}\right)^{\mathbf{b}}$ \\
\hline $\mathbf{4 a}$ & $2-\mathrm{Cl}$ & 81 & $120-121$ \\
\hline $\mathbf{4 b}$ & $3-\mathrm{Cl}$ & 75 & $131-134$ \\
\hline $\mathbf{4 c}$ & $4-\mathrm{Cl}$ & 75 & $156-158$ \\
\hline $\mathbf{4 d}$ & $2-\mathrm{F}$ & 73 & $120-122$ \\
\hline $\mathbf{4 e}$ & $3-\mathrm{F}$ & 74 & $142-145$ \\
\hline $\mathbf{4 f}$ & $4-\mathrm{F}$ & 76 & $153-155$ \\
\hline $\mathbf{4 g}$ & $2-\mathrm{NO}_{2}$ & 76 & $158-163$ \\
\hline $\mathbf{4 h}$ & $3-\mathrm{NO}_{2}$ & 85 & $147-150$ \\
\hline $\mathbf{4 i}$ & $4-\mathrm{NO}_{2}$ & 90 & $99-101$ \\
\hline $\mathbf{4 j}$ & $2-\mathrm{OCH}_{3}$ & 84 & $127-130$ \\
\hline $\mathbf{4 k}$ & $3-\mathrm{OCH}_{3}$ & 81 & $118-120$ \\
\hline $\mathbf{4 l}$ & $4-\mathrm{OCH}_{3}$ & 80 & $142-144$ \\
\hline $\mathbf{4 m}$ & $3-\mathrm{OH}$ & 74 & $157-160$ \\
\hline $\mathbf{4 n}$ & $4-\mathrm{OH}$ & 65 & $183-186$ \\
\hline $\mathbf{4 0}$ & $2,4-\mathrm{OCH}_{3}$ & 47 & oil \\
\hline $\mathbf{4 p}$ & $3,4-\mathrm{OCH}_{3}$ & 74 & $72-75$ \\
\hline $\mathbf{4 q}$ & $2,3-\mathrm{OCH}_{3}$ & 60 & $93-95$ \\
\hline $\mathbf{4 r}$ & $2,5-\mathrm{OCH}_{3}$ & 58 & oil \\
\hline
\end{tabular}

\section{CONCLUSION}

In summary, this work showed the synthesis of eighteen new 3-(benzo[1,3]dioxol-5-yl)-2phenylthiazolidin-4-ones from both electron-release and electron-withdraw substituted arenealdehydes. In the next step, these compounds will be submitted to biological studies.

\section{ACKNOWLEDGEMENTS}

The authors thanks: CAPES, FAPERGS and UFPel

\section{REFERENCES}

${ }^{1}$ Jain, A. K.; Vaidya, A.; Ravichandran, V.; Kashaw, S.K.; Agrawal, R.K. Bioorg. Med. Chem. 2012, 20, 3378.

${ }^{2}$ O. Guzel, A. Salman, J. Med. Chem. 2009, 24, 1015.

${ }^{3}$ R. Ottanà, R. Maccari, M. Giglio, A. Del Corso, M. Cappiello, U. Mura, S. Cosconati, L. Marinelli, E. Novellino, S. Sartini, C. La Motta, F. Da Settimo, Eur. J.Med. Chem. 2011, 4646, 2797.

${ }^{4}$ Vintonyak, V.V.; Warburg, K.; Over, B.; Hubel, K.; Rauh, D.; Waldmann, $\mathrm{H}$.; Tetrahedron 2011,67, 6713 .

${ }^{5}$ R.K. Rawal, S.B. Katti, N. Kaushik-Basu, P. Arora, Z. Pan, Bioorg. Med. Chem. Lett.2008, 18, 6110.

${ }^{6}$ Cunico, W.; Gomes, C. R. B.; Vellasco, W. T., Jr. Mini-Rev. Org. Chem. 2008, 5, 336 . 